\title{
The use of antibiotic-loaded bone cement does not increase antibiotic resistance after primary total joint arthroplasty
}

\author{
Kaspar Tootsi $^{1,2,3} \cdot$ Victoria Heesen $^{1} \cdot$ Martin Lohrengel ${ }^{1} \cdot$ Andreas Eugen Enz $^{4} \cdot$ Sebastian Illiger ${ }^{1}$. \\ Wolfram Mittelmeier ${ }^{4}$. Christoph H. Lohmann ${ }^{1}$
}

Received: 23 April 2021 / Accepted: 21 June 2021 / Published online: 9 July 2021

(c) The Author(s) 2021

\begin{abstract}
Purpose One of the preventive strategies for periprosthetic joint infection (PJI) is the use of antibiotic-loaded bone cement (ALBC) in primary total joint arthroplasty (TJA). Even though it is widely used, there are concerns about the development of antibacterial resistance. The aim of the study was to investigate whether using ALBC in primary TJA increases the antibioticresistant PJI. The hypothesis was that the regular use of ALBC does not increase the rate of resistant PJI.

Methods Patients with confirmed PJI who had revision surgery from year 2010 to 2019 were included in this international multicenter study. The ALBC group was compared to the non-ALBC TJA group from the same time period. Medical records were used to collect clinical (age, gender, body mass index, comorbidities), TJA-related (type of operation, implant type and survival) and PJI-related (cultured microorganism, antibiogram) data. Resistance to gentamicin, clindamycin and vancomycin were recorded from the antibiograms. Multiple logistic regression model was used to identify risk factors and account for the potential confounders.

Results 218 patients with PJI were included in the study: 142 with gentamicin-loaded bone cement and 76 in the non-ALBC group. The average age in the ALBC group was $71 \pm 10$ years and $62 \pm 12$ years in the comparison group $(p<0.001)$. Coagulase negative Staphylococci (CONS) were the most common (49\%) isolated pathogens. The use of ALBC did not increase the rate of any resistant bacteria significantly $(\mathrm{OR}=0.79(0.42-1.48), p=0.469)$. The presence of CONS was associated with higher risk of antibiotic resistance.

Conclusions The current study demonstrates no increase in antibiotic resistance due to ALBC after primary TJA. Thus, the use of ALBC during primary TJA should not be feared in the context of antimicrobial resistance.
\end{abstract}

Level of evidence III.

Keywords Antimicrobial resistance $\cdot$ Antibiotic-loaded cement $\cdot$ Arthroplasty $\cdot$ Periprosthetic joint infection · Gentamicin · Clindamycin

Christoph H. Lohmann

christoph.lohmann@med.ovgu.de

1 Department of Orthopaedics, Otto-Von-Guericke University, Leipziger Str. 44, 39120 Magdeburg, Germany

2 Department of Traumatology and Orthopaedics, University of Tartu, Puusepa Str 8, 51014 Tartu, Estonia

3 Department of Orthopaedics, Tartu University Hospital, Puusepa Str 8, 51014 Tartu, Estonia

4 Klinik und Poliklinik für Orthopädie, Universitätsmedizin Rostock, Doberaner Straße 142, 18057 Rostock, Germany

\section{Introduction}

The widespread use of ALBC in various countries has raised concerns about the development of antibiotic resistance in patients with PJI [2, 29, 32]. Two different levels of antibiotics in the cement are differentiated: a low dose $(<2 \mathrm{~g}$ in $40 \mathrm{~g}$ of cement) for prophylactic use in primary arthroplasty and high concentration for infection treatment [5]. The prophylactic concentration of antibiotics in ALBC has been shown to be inconsequential to the mechanical properties of polymethylmethacrylate (PMMA) bone cement [13]. The use of ALBC enables to achieve high levels of antibiotics locally, while causing less systemic side-effect. Regardless of the benefits, several concerns have been raised for using ALBC 
as a routine practice that include potential increase in antibiotic resistance and decreased mechanical strength of bone cement $[3,9]$. Unfortunately, clinical research investigating the benefits and risks of ALBC are limited and have failed to reach a uniform conclusion, therefore, the routine clinical practice varies hugely [31]. Several studies have shown decreased rates of surgical site infection for using ALBC, which is why it is a widely spread routine clinical practice, especially in Europe [10, 17, 35].

The growth of drug-resistant bacteria in PJI is concerning and the drastic rise of overall prevalence of PJI calls for interventions lowering the infection rate [2,22]. The use of ALBC requires specific attention for determining the longterm risk of inducing antibacterial resistance. Hope et al. have published an increased prevalence up to $30 \%$ of gentamicin resistant bacteria in revision TJA after prior exposure to ALBC [15]. Other larger analysis found no significant difference [12]. Therefore, the data are limited, based on small single-center studies and contradictory, thus larger studies including data from multinational centers is highly needed.

The aim of the present study was to determine whether the use of ALBC is associated with higher prevalence of antibiotic-resistant bacteria as a cause of PJI after primary total knee or hip joint replacement. Even low dose of antibiotics can potentially cause resistance due to not eradicating all of the bacteria and enabling the bacteria to develop mechanisms to counteract the antibiotics. The hypothesis of the present study was that using ALBC in primary TJA does not increase antibacterial resistance. Therefore, the first revisions of cemented or cementless primary total hip arthroplasties (THAs) or total knee arthroplasties (TKAs) were analyzed for occurrence of resistant bacteria in an international multicenter study. Thorough information about the bacterial susceptibility to clinically most relevant antibiotics (gentamicin, clindamycin and vancomycin) was gathered and analyzed.

\section{Methods}

This multicenter study includes patients presenting with a knee or hip joint PJI. The study participants were recruited retrospectively from three university hospitals. Prerequisite for inclusion was no intraarticular surgery of the respective joint prior to the index surgery. A total of 218 patients met the inclusion and exclusion criteria during the study period and were included in the analysis. $62 \%$ of the study participants had their primary operation in one of the three study centers and the remaining $38 \%$ were initially operated somewhere else.

The study includes patients with confirmed PJI ( $\geq 2$ positive cultures) who underwent revision surgery from
December 2010 to December 2019. The resistograms were analyzed for any resistant bacteria, difficult to treat bacteria, particularly for resistance to gentamicin, since it was the only antibiotic added to the bone cement during primary TJA in these hospitals in the study period. The data were acquired from the hospital's local database.

Patients with no prior implant-associated infection who were eligible for revision surgery were included in the study. Furthermore, only patients having at least two positive microbiological cultures with antibiogram were included.

Patients were excluded from the study if they had had any intraarticular surgery prior to the primary TJA in the respective joint or prior surgical revision of the respective joint. If a sepsis was documented prior to revision surgery or if there was missing documentation of the used bone cement, the patients were also excluded.

The antibiotic agent used in the ALBC group was gentamicin with different premixed formulations: Palacos $R+G$ ( $0.5 \mathrm{~g}$ of gentamicin in $40 \mathrm{~g}$ of bone cement) (Heraeus Medical GmbH, Germany), Cemex Genta (1.0 g/40 g) (Merete $\mathrm{GmbH}$, Germany), Optipac Refobacin R $(0.5 \mathrm{~g} / 40 \mathrm{~g})$ and Biomet R (0 g/40) (Zimmer Biomet Holdings, Inc., USA), Smartset GHV (1.0 g/40 g) and Depuy CMW (1 g/40 g) (DePuy Synthes, Johnson \& Johnson Corporation, USA). The comparison group comprised of cementless and cemented without the ALBC PJI revision cases from the same three hospitals. The same inclusion criteria applied for the non-ALBC group, except for the use of ALBC during the primary TJA.

During revision surgery, at least five tissue cultures were obtained. Tissues were transferred to the hospital's Department of Microbiology within $30 \mathrm{~min}$ after retrieving the tissue samples from the infected joints to ensure diagnostic quality, particularly with respect to anaerobic bacteria. The resistograms and specific antimicrobial resistance of the bacteria were obtained after culture for 2 weeks. Resistance to gentamicin, clindamycin and vancomycin were assessed according to the EUCAST guidelines [18]. Proven PJI was considered when at least two samples were positive for bacteria.

Early PJI was defined as symptom development within the first 6 weeks from the primary TJA and symptom development $>6$ weeks after implantation was defined as a late infection. Charlson comorbidity index and American Society of Anaesthesiologists (ASA) score was used to describe the comorbidities within the study groups [7]. Survival of the implants was measured from the primary TJA to the revision surgery in years.

The study has been approved by the local Ethics Committees (Otto von Guericke University, No 106/17; Rostock University Medical Center, No A2020-0297; University of Tartu, No 330/T-20). 
Table 1 General parameters of antibiotic-loaded cement (ALBC) group and the nonALBC group with periprosthetic joint infection

\begin{tabular}{lllr}
\hline Variable & $\begin{array}{l}\text { ALBC } \\
(n=142)\end{array}$ & $\begin{array}{l}\text { Non-ALBC } \\
(n=76)\end{array}$ & $p$ value \\
\hline Age (years) & $71 \pm 10$ & $62 \pm 12$ & $<0.001$ \\
Male/female & $72 / 70$ & $54 / 22$ & 0.006 \\
THA/TKA $(n)$ & $50 / 92$ & $64 / 12$ & $<0.001$ \\
BMI $\left(\mathrm{kg} / \mathrm{m}^{2}\right)$ & $31.3 \pm 6.5$ & $30.3 \pm 6.6$ & 0.250 \\
ASA score & $2.7 \pm 0.5$ & $2.4 \pm 0.5$ & 0.005 \\
Charlson comorbidity index & $4.9 \pm 2.9$ & $3.4 \pm 2.3$ & $<0.001$ \\
Early/late infections $(n)$ & $32 / 110$ & $18 / 58$ & 0.866 \\
Implant survival (years) & $3.7 \pm 5.6$ & $4.9 \pm 9.8$ & 0.303 \\
Primary implantation year (mean & $2012(1984-2019)$ & $2012(1993-2019)$ & 0.250 \\
$\quad$ (range) & & & \\
\hline
\end{tabular}

$A L B C$ antibiotic-loaded bone cement, THA total hip arthroplasty, TKA total knee arthroplasty, $B M I$ body mass index, ASA American Society of Anaesthesiologists
Table 2 Type of fixation of primary implant

\begin{tabular}{lrc}
\hline & $n$ & Percent \\
\hline Cementless THA & 59 & 27.1 \\
Hybrid THA & 23 & 10.6 \\
Cemented THA & 32 & 14.7 \\
Cementless TKA & 9 & 4.1 \\
Hybrid TKA & 13 & 6.0 \\
Cemented TKA & 82 & 37.6 \\
Total & 218 & 100 \\
\hline
\end{tabular}

THA total hip arthroplasty, TKA total knee arthroplasty

\section{Statistical analysis}

Data are presented as mean $\pm \mathrm{SD}$. The Chi-square test or Fischer's exact test were used to compare group proportions when appropriate. Student's $t$ test or Mann-Whitney $U$ test were used for group comparisons depending on the normality of the distribution of the data. Logistic regression analysis was used to include the potential confounding factors in the analysis. Statistical analysis of the data was performed by SPSS 26.0 (SPSS Inc., Chicago IL, USA) for Windows.

\section{Results}

The general parameters of the study population are presented in Table 1. There were 142 participants in the ALBC group and 76 in the non-ALBC. The study includes 50 early and 168 late infections. The proportion of early infections, average implant survival and body mass index (BMI) did not differ significantly between the groups (Table 1). The type of primary TJA and the implant are presented in Tables 2 and 3 , respectively.

Approximately half of the infections (48.6\%) were caused by coagulase negative Staphylococci (CONS)
Table 3 Type of primary implant

\begin{tabular}{lrl}
\hline & $n$ & Percent \\
\hline Standard THA & 110 & 50.5 \\
Short hip stem & 1 & 0.5 \\
Surface replacement THA & 1 & 0.5 \\
Proximal femoral replacement & 2 & 0.9 \\
Surface replacement TKA & 94 & 43.1 \\
Hinged TKA & 8 & 3.7 \\
Distal femoral replacement & 2 & 0.9 \\
Total & 218 & 100 \\
\hline
\end{tabular}

THA total hip arthroplasty, TKA total knee arthroplasty

(Table 4). The second most prevalent isolated pathogen was Staphylococcus aureus in $34.4 \%$ of the cases. The prevalence of the different isolated bacteria is presented in Table 4. Polymicrobial infection was identified in $16 \%$ of the study participants. The overall prevalence of bacteria with any recorded antibiotic resistance was $36.5 \%$ (76 of the 218 patients).

Table 5 demonstrates the proportions of resistant bacteria in each study group. There was no significant correlation for the use of ALBC and having any resistant bacteria $(p=0.658)$. Also, there were no significant differences for gentamicin or clindamycin resistance between the study groups.

To identify variables associated with the antibiotic resistance and account for the differences in clinical parameters between the study groups, a logistic regression analysis was performed (Table 6). The model revealed that the use of ALBC in the primary TJA was not associated with the prevalence of resistant bacterial strains. However, the presence of CONS was significantly associated with antibiotic resistance. A model investigating the determinants of pure gentamicin resistance found no significant effect of the use of ALBC (data not shown). 
Table 4 List and proportion of pathogens isolated from periprosthetic joint infections

\begin{tabular}{|c|c|c|c|}
\hline Isolated pathogen & $\begin{array}{l}\text { ALBC } \\
\text { group } \\
(n)\end{array}$ & $\begin{array}{l}\text { Non- } \\
\text { ALBC } \\
\text { group }(n)\end{array}$ & Total $(\%)$ \\
\hline CONS & 71 & 35 & 48.6 \\
\hline Staphylococcus epidermidis & 50 & 23 & 33.5 \\
\hline Staphylococcus capitis & 3 & 5 & 3.7 \\
\hline Staphylococcus hominis & 4 & 2 & 2.8 \\
\hline Staphylococcus lugdunensis & 3 & 2 & 2.3 \\
\hline Staphylococcus warneri & 2 & 1 & 1.4 \\
\hline Staphylococcus haemolyticus & 2 & 1 & 1.8 \\
\hline Staphylococcus pasteuri & 1 & 0 & 0.5 \\
\hline Staphylococcus caprae & 2 & 0 & 0.9 \\
\hline Staphylococcus simulans & 2 & 0 & 0.9 \\
\hline Staphylococcus vitulinus & 0 & 1 & 0.5 \\
\hline Staphylococcus oralis & 1 & 0 & 0.5 \\
\hline Staphylococcus aureus & 49 & 26 & 34.4 \\
\hline Enterococcus faecalis & 11 & 4 & 6.9 \\
\hline Propionibacterium acnes/avidum & 1 & 5 & 2.8 \\
\hline Escherichia coli & 2 & 4 & 2.8 \\
\hline Pseudomonas aeruginosa & 13 & 0 & 1.4 \\
\hline Bacteroides fragilis & 0 & 1 & 0.5 \\
\hline Finegoldia magna & 1 & 0 & 0.5 \\
\hline Corynebacterium amycolatum & 1 & 0 & 0.5 \\
\hline Micrococcus luteus & 1 & 0 & 0.5 \\
\hline $\begin{array}{l}\text { Cornybacterium tuberculostear- } \\
\text { ium }\end{array}$ & 0 & 1 & 0.5 \\
\hline Trueperella bernardiae & 1 & 0 & 0.5 \\
\hline Streptococcus agalactiae & 4 & 2 & 2.8 \\
\hline Bacillus spp & 0 & 4 & 1.8 \\
\hline Pseudomonas stutzeri & 1 & 0 & 0.5 \\
\hline Streptococcus pyogenes & 1 & 1 & 0.9 \\
\hline Bacillus pumilus & 0 & 1 & 0.5 \\
\hline Citrobacter koseri & 0 & 1 & 0.5 \\
\hline Acinetobacter haemolyticus & 0 & 1 & 0.5 \\
\hline Bacillus cereus & 0 & 1 & 0.5 \\
\hline Acinetobacter schindleri & 1 & 0 & 0.5 \\
\hline Streptococcus dysgalactiae & 4 & 4 & 3.7 \\
\hline Streptococcus anginosus & 1 & 1 & 0.9 \\
\hline Streptococcus salivarius & 1 & 0 & 0.5 \\
\hline Candida parapsi & 1 & 0 & 0.5 \\
\hline Enterobacter cloacae & 1 & 0 & 0.5 \\
\hline Listeria monocytogenes & 1 & 0 & 0.5 \\
\hline Streptococcus gordonii & 1 & 0 & 0.5 \\
\hline Parvimonas micra & 1 & 0 & 0.5 \\
\hline
\end{tabular}

CONS coagulase negative Staphylococci

\section{Discussion}

The most important finding of the study was the absence of a significant increase of antibiotic resistance for using
ALBC. In the present study, a group of 218 patients with PJI undergoing revision surgery was examined to determine whether the use of ALBC for primary TJA increased the rate of resistant bacteria. The results demonstrate no significant increase in the prevalence of resistant bacteria after using ALBC in the primary TJA. Interestingly, positive CONS culture was significantly associated with the prevalence of resistant strains.

PJI is among the most devastating complications of TJA that can lead to repeated surgeries, poor functional outcome and even amputation of the limb and death [19]. Even though the rate of PJI is low $(<1 \%$ in most of the high-volume arthroplasty centers), the steady increase in the absolute number of TJA leads to increase in PJI $[6,21]$. Therefore, prevention of PJI is of utmost importance.

Different strategies have been applied to prevent PJI. The use of intravenous preoperative antibiotics is among the most effective approaches. Adding antibiotics to the bone cement in addition to intravenous use is an effective prevention method that has shown to decrease the PJI rate in several high-quality studies up to $50 \%[8,10,26]$. Gentamicin and clindamycin are the most often added antibiotics to the bone cement [38]. Low-dose mixture $(<2 \mathrm{~g}$ per $40 \mathrm{~g}$ of bone cement) is used for prevention in primary TJA. ALBC has been shown to be associated with lower rate of PJI in primary TJA, however, it is still not a routine practice worldwide, because of higher cost and safety concerns [16].

Three main problems may be associated with the safety of routine use of ALBC: decreased mechanical strength of the cement, local and systemic toxicity, and induction of antibiotic-resistant bacterial strains. Nevertheless, low levels of added antibiotics to the ALBC that is optimal for primary TKA and THA have not been demonstrated to cause negative clinical consequences $[13,35]$. Gentamicin levels of $\leq 1 \mathrm{~g}$ in $40 \mathrm{~g}$ of bone cement that are mostly used in primary TJA, do not affect the mechanical strength [9]. Also, the cytotoxicity of ALBC has not been established to be clinically relevant [35]. Therefore, currently the most important safety issue is the possible induction of antibiotic-resistant bacterial strains, which is the main focus of the present study.

The length of antibiotics release from ALBC is enough to cover the perioperative period of TJA, however, after several weeks, the concentration falls to subtherapeutic levels, which might promote the development of antibiotic resistance [11]. Elution characteristics of the ALBC may be different depending on effective joint space (enlarged in loosened TJA's), implant surface area and different joints. However, the direct effects of eluted antibiotics from bone cements on bacteria follow similar principles and the initial burst release from the ALBC is sufficient to eradicate the bacteria and not cause resistance. In addition, the development of resistance might also be associated with the biomaterial properties of bone cement $[1,20,36]$. 
Table 5 Proportions of antibiotic resistance in the antibiotic-loaded bone cement and in the non-ALBC arthroplasty groups

\begin{tabular}{lccc}
\hline & $\begin{array}{l}\text { ALBC } \\
(n=142)\end{array}$ & $\begin{array}{l}\text { Non-ALBC } \\
(n=76)\end{array}$ & $p$ value \\
\hline Any resistant bacteria $(n(\%))$ & $48(34)$ & $28(37)$ & 0.658 \\
Resistant bacteria to gentamicin $(n(\%))$ & $27(19)$ & $7(9)$ & 0.076 \\
Resistant bacteria to clindamycin $(n(\%))$ & $26(19)$ & $18(24)$ & 0.381 \\
Resistant bacteria to vancomycin $(n(\%))$ & $3(2)$ & $1(1)$ & 0.860 \\
\hline
\end{tabular}

$A L B C$ antibiotic-loaded bone cement
Table 6 Logistic regression analysis showing no significant predictors for having antibiotic-resistant bacteria

\begin{tabular}{lrrrl}
\hline & \multicolumn{1}{c}{$B$} & OR & CI (95\%) & $p$ value \\
\hline ALBC & -0.24 & 0.79 & $0.43-1.48$ & 0.469 \\
Charlson comorbidity index & 0.03 & 1.03 & $0.92-1.15$ & 0.573 \\
Implant survival in years & -0.06 & 0.94 & $0.88-1.00$ & 0.060 \\
Presence of CONS & 0.90 & 2.46 & $1.38-4.39$ & 0.002 \\
\hline
\end{tabular}

$O R$ odds ratio, $C I$ confidence interval, $A L B C$ antibiotic-loaded bone cement, CONS coagulase negative Staphylococci

Several mechanisms have been described, how resistance to antibiotics is developed. De novo mutations of previously susceptible bacteria are most probably the leading cause of resistance [23]. The use of antibiotics will provide a competitive advantage to the resistant mutated bacteria. Also, the existing bacteria resistant to antibiotics may simply be selected out in an antibiotic rich environment [38]. Furthermore, resistance genes can be spread between different strains of bacteria through plasmids of chromosomal inserts (via bacteriophages and transposons) [23].

The present study demonstrated that using ALBC in primary TKA and THA did not increase the prevalence of antibiotic-resistant bacterial PJI in the present study's cohort. This suggests that other factors than ALBC might be more importantly impacting the spread of antibiotic resistance. Interestingly, the presence of CONS was associated with increased rate of resistant bacteria (Table 6). CONS are part of normal skin flora that can cause infection in immunocompromised patients and in patients with large foreign bodies (e.g. arthroplasty implants). The spread of resistant CONS is promoted by the use of antibiotics in the hospitals that provides a reservoir-resistant bacteria. Even though the origin of the infectious bacteria is unknown, the hospitals are one of the most probable places.

Other possible factors include: (1) inappropriate prescription or application of antibiotics, (2) high and increasing prevalence of multi-drug-resistant bacteria in the hospital, (3) small number of new and effective antimicrobials to efficiently eradicate resistant strains, (4) widespread agricultural use of antibiotics leading natural selection towards resistant strains that are transferred to humans through food supply [24, 25, 34, 37].

The results of the present study indicating no increased antibiotic resistance due to ALBC are supported by several studies $[9,12]$. However, some published data suggest a significantly higher prevalence of antibiotic-resistant bacteria with ALBC $[8,14,33]$. The largest analysis so far from the UK registry data found that the use of ALBC may increase the resistance to gentamicin [14]. However, that study only looked at Staphylococcal infections [14]. Even though the Staphylococci are the largest group as the cause of PJI, a sizable proportion of infections are caused by other microorganisms (33\% in the current study) that were not included in the UK cohort. Thus, the present study provides a significant novel contribution by including detailed data about the whole spectrum of pathogens in PJI.

The most prevalent pathogens isolated from the PJI in the present study were CONS (mostly S. epidermidis) and Staphylococcus aureus that is consistent with previous research [2, 25, 27, 33]. However, the rate of polymicrobial infections is substantially higher (16\%) in the present study compared with previously reported rates of $6 \%$ [33]. The difference might occur due to a genuine shift towards more pathogens in PJI, however, there are also some methodological considerations: the present study is more recent and the sample collecting and the microbiology culture methods might have changed during the time period (ca 10 years) to be able to detect more pathogens.

The present study has various limitations: the retrospective nature does not allow to confirm cause and effect. Other risk factors for developing resistance that were not included in the analysis might confound the effect of ALBC. Several different premixed ALBC-s with varying elution properties were used in the study that might have a confounding effect on the development of antibacterial resistance. Furthermore, the origin of the bacteria that caused the PJI is not known in the present study. The control group consists mainly of cementless TJA-s that might introduce a bias due to the absence of bone cement in the non-ALBC group. Nevertheless, the study population is thoroughly described compared with registry studies, all cultured microorganisms from the PJI samples have been included in the analysis, the bacteria have been identified on species level and the study entails 
resistance information about clinically most relevant antibiotics (gentamicin, clindamycin and vancomycin).

Current evidence together with the results of the present study suggest that the use of ALBC is a safe and effective method for preventing PJI and should be encouraged.

\section{Conclusion}

The present study demonstrates that the standard use ALBC for the fixation of primary TJA does not increase the prevalence of antibacterial resistance. Having CONS as the causative agent of PJI is associated with increased risk for antibiotic resistance.

Acknowledgements The authors are grateful to dr Julia Reinvald and dr Egon Puuorg (Department of Orthopaedics, Tartu University Hospital, Estonia) for the help in data collection.

Funding Open Access funding enabled and organized by Projekt DEAL. This work was supported by the European Union's Seventh Framework Programme (FP7/2007-2013) (grant agreement no.:602398), by grants from the Estonian Research Council (PSG418 and PSG610), by the University of Tartu Foundation (Elmerice and Andreas Traks scholarship) and DAAD short-term research grant.

\section{Declarations}

Conflict of interest The authors declare that they have no conflict of interest.

Ethical approval The study has been approved by the local Ethics Committees (Otto von Guericke University, No 106/17; Rostock University Medical Center, No A2020-0297; University of Tartu, No 330/T-20).

Open Access This article is licensed under a Creative Commons Attribution 4.0 International License, which permits use, sharing, adaptation, distribution and reproduction in any medium or format, as long as you give appropriate credit to the original author(s) and the source, provide a link to the Creative Commons licence, and indicate if changes were made. The images or other third party material in this article are included in the article's Creative Commons licence, unless indicated otherwise in a credit line to the material. If material is not included in the article's Creative Commons licence and your intended use is not permitted by statutory regulation or exceeds the permitted use, you will need to obtain permission directly from the copyright holder. To view a copy of this licence, visit http://creativecommons.org/licenses/by/4.0/.

\section{References}

1. Arciola CR, Campoccia D, Montanaro L (2002) Effects on antibiotic resistance of Staphylococcus epidermidis following adhesion to polymethylmethacrylate and to silicone surfaces. Biomaterials 23(6):1495-1502

2. Benito N, Franco M, Ribera A, Soriano A, Rodriguez-Pardo D, Sorlí L et al (2016) Time trends in the aetiology of prosthetic joint infections: a multicentre cohort study. Clin Microbiol Infect 22(8):732.e1-732.e8

3. Berberich C, Sanz-Ruiz P (2019) Risk assessment of antibiotic resistance development by antibiotic-loaded bone cements: is it a clinical concern? EFORT Open Rev 4(10):576-584

4. Best JT (2005) Revision total hip and total knee arthroplasty. Orthop Nurs 24(3):174-179-181

5. Bistolfi A, Massazza G, Verné E et al (2011) Antibiotic-loaded cement in orthopedic surgery: a review. ISRN Orthop. https://doi. org/10.5402/2011/290851

6. Chang CH, Lee SH, Lin YC, Wang YC, Chang CJ, Hsieh PH (2020) Increased periprosthetic hip and knee infection projected from 2014 to 2035 in Taiwan. J Infect Public Health 13(11):1768-1773

7. Charlson ME, Pompei P, Ales KL, MacKenzie CR (1987) A new method of classifying prognostic comorbidity in longitudinal studies: development and validation. J Chronic Dis 40(5):373-383

8. Chiu FY, Chen CM, Lin CF, Lo WH (2002) Cefuroxime-impregnated cement in primary total knee arthroplasty: a prospective, randomized study of three hundred and forty knees. J Bone Joint Surg Am 84(5):759-762

9. Dunne NJ, Hill J, McAfee P, Kirkpatrick R, Patrick S, Tunney M (2008) Incorporation of large amounts of gentamicin sulphate into acrylic bone cement: effect on handling and mechanical properties, antibiotic release, and biofilm formation. Proc Inst Mech Eng 222(3):355-365

10. Engesaeter LB, Lie SA, Espehaug B, Furnes O, Vollset SE, Havelin LI (2003) Antibiotic prophylaxis in total hip arthroplasty: effects of antibiotic prophylaxis systemically and in bone cement on the revision rate of 22,170 primary hip replacements followed 0-14 years in the Norwegian Arthroplasty Register. Acta Orthop Scand 74(6):644-651

11. Fink B, Vogt S, Reinsch M, Büchner H (2011) Sufficient release of antibiotic by a spacer 6 weeks after implantation in two-stage revision of infected hip prostheses. Clin Orthop 469(11):3141-3147

12. Hansen EN, Adeli B, Kenyon R, Parvizi J (2014) Routine use of antibiotic laden bone cement for primary total knee arthroplasty: impact on infecting microbial patterns and resistance profiles. $\mathbf{J}$ Arthroplasty 29(6):1123-1127

13. Hanssen AD (2004) Prophylactic use of antibiotic bone cement: an emerging standard-in opposition1. J Arthroplasty 19(4):73-77

14. Holleyman RJ, Deehan DJ, Walker L, Charlett A, Samuel J, Shirley MDF et al (2019) Staphylococcal resistance profiles in deep infection following primary hip and knee arthroplasty: a study using the NJR dataset. Arch Orthop Trauma Surg 139(9):1209-1215

15. Hope PG, Kristinsson KG, Norman P, Elson RA (1989) Deep infection of cemented total hip arthroplasties caused by coagulasenegative staphylococci. J Bone Joint Surg Br 71(5):851-855

16. Hoskins T, Shah JK, Patel J, Mazzei C, Goyette D, Poletick E et al (2020) The cost-effectiveness of antibiotic-loaded bone cement versus plain bone cement following total and partial knee and hip arthroplasty. J Orthop 20:217-220

17 Jämsen E, Huhtala H, Puolakka T, Moilanen T (2009) Risk factors for infection after knee arthroplasty. A register-based analysis of 43,149 cases. J Bone Joint Surg Am 91(1):38-47

18. Kahlmeter G, Brown DF, Goldstein FW et al (2003) European harmonization of MIC breakpoints for antimicrobial susceptibility testing of bacteria. J Antimicrob Chemother 52(2):145-148

19. Kandel CE, Jenkinson R, Daneman N et al (2019) Predictors of treatment failure for hip and knee prosthetic joint infections in the setting of 1- and 2-stage exchange arthroplasty: a multicenter retrospective cohort. Open Forum Infect Dis 6(11):452 
20. Kendall RW, Duncan CP, Smith JA, Ngui-Yen JH (1996) Persistence of bacteria on antibiotic loaded acrylic depots. A reason for caution. Clin Orthop Relat Res 329:273-280

21. Kurtz SM, Lau E, Watson H, Schmier JK, Parvizi J (2012) Economic burden of periprosthetic joint infection in the United States. J Arthroplasty 27(8 Suppl):61-5.e1

22. Lenguerrand E, Whitehouse MR, Beswick AD, Jones SA, Porter ML, Blom AW (2017) Revision for prosthetic joint infection following hip arthroplasty. Bone Jt Res 6(6):391-398

23. Livermore DM (2000) Epidemiology of antibiotic resistance. Intensive Care Med 26(Suppl 1):S14-S21

24. Nagvekar V, Sawant S, Amey S (2020) Prevalence of multi drug resistant Gram-negative bacteria cases at admission in multispecialty hospital. J Glob Antimicrob Resist 22:457-461

25. Nickinson RSJ, Board TN, Gambhir AK, Porter ML, Kay PR (2010) The microbiology of the infected knee arthroplasty. Int Orthop 34(4):505-510

26. Parvizi J, Saleh KJ, Ragland PS, Pour AE, Mont MA (2008) Efficacy of antibiotic-impregnated cement in total hip replacement. Acta Orthop 79(3):335-341

27. Peersman G, Laskin R, Davis J, Peterson M (2001) Infection in total knee replacement: a retrospective review of 6489 total knee replacements. Clin Orthop Relat Res 392:15-23

28. Pulido L, Ghanem E, Joshi A, Purtill JJ, Parvizi J (2008) Periprosthetic joint infection: the incidence, timing, and predisposing factors. Clin Orthop Relat Res 466(7):1710-1715

29. Ravi S, Zhu M, Luey C, Young SW (2016) Antibiotic resistance in early periprosthetic joint infection. ANZ J Surg 86(12):1014-1018

30. Schmitt DR, Killen C, Murphy M, Perry M, Romano J, Brown N (2020) The impact of antibiotic-loaded bone cement on antibiotic resistance in periprosthetic knee infections. Clin Orthop Surg 12(3):318-323

31. Schwarz EM, Parvizi J, Gehrke T, Aiyer A, Battenberg A, Brown SA et al (2019) 2018 International Consensus Meeting on
Musculoskeletal Infection: Research Priorities from the General Assembly Questions. J Orthop Res 37(5):997-1006

32. Siljander MP, Sobh AH, Baker KC, Baker EA, Kaplan LM (2018) Multidrug-resistant organisms in the setting of periprosthetic joint infection-diagnosis, prevention, and treatment. J Arthroplasty 33(1):185-194

33. Stefánsdóttir A, Johansson D, Knutson K, Lidgren L, Robertsson O (2009) Microbiology of the infected knee arthroplasty: report from the Swedish Knee Arthroplasty Register on 426 surgically revised cases. Scand J Infect Dis 41(11-12):831-840

34. Struelens MJ (1998) The epidemiology of antimicrobial resistance in hospital acquired infections: problems and possible solutions. BMJ 317(7159):652-654

35. Sultan AA, Samuel LT, Umpierrez E et al (2019) Routine use of commercial antibiotic-loaded bone cement in primary total joint arthroplasty: a critical analysis of the current evidence. Ann Transl Med 7(4):73

36. Thomes B, Murray P, Bouchier-Hayes D (2002) Development of resistant strains of Staphylococcus epidermidis on gentamicinloaded bone cement in vivo. J Bone Joint Surg Br 84(5):758-760

37. Ventola CL (2015) The antibiotic resistance crisis. Pharm Ther 40(4):277-283

38. Walker LC, Baker P, Holleyman R, Deehan D (2017) Microbial resistance related to antibiotic-loaded bone cement: a historical review. Knee Surg Sports Traumatol Arthrosc 25(12):3808-3817

Publisher's Note Springer Nature remains neutral with regard to jurisdictional claims in published maps and institutional affiliations. 